\title{
New Classification of Crown Forms and Gingival Characteristics in Taiwanese
}

\author{
Yu-Hsiang Chou ${ }^{1}$, Chi-Cheng Tsai ${ }^{1, *}$, Jen-Chyan Wang ${ }^{1}$, Ya-Ping $\mathrm{Ho}^{1}$, Kun-Yen $\mathrm{Ho}^{1}$ and \\ Chuen-Chyi Tseng ${ }^{2, *}$
}

\begin{abstract}
${ }^{I}$ Department of Periodontics, Chung-Ho Memorial Hospital, Kaohsiung Medical University, 100 Shih-Chuan 1st Road, Kaohsiung 807, Taiwan and ${ }^{2}$ Department of Periodontics, Chi-Mei Medical Center, 901 Chung Hwa Road, Yong Kang City Tainan Taiwan
\end{abstract}

\begin{abstract}
Objectives: The aim of the investigation was to examine the forms of the crowns in the maxillary anterior tooth segment and corresponding gingival characteristics among healthy Taiwanese subjects.

Materials and Methods: The crown width at the apical third (CW), length (CL), gingival angle (GA) and the interdental papilla height were assessed from the diagnostic stone model using a calibrated periodontal caliper. A CW/CL-ratio was calculated for each tooth and averaged for each tooth region. Gingival thickness (GT) and width of keratinized gingiva (WG) were measured clinically.

Results: The cluster analysis revealed 3 classifications of crown forms: narrow (N), compound (C) and square (S) types. There was a significant difference among the 3 classifications with respect to CW/CL-ratio, GT, and WG ( $\mathrm{p}<0.0001$ ).

Conclusions: The results demonstrated varied crown forms and corresponding gingival characteristics in Caucasian and Taiwanese. The new classifications hinted that there was a polymorphism in different races and could be a valuable esthetic guideline and reference for anterior tooth rehabilitation, including various periodontal and restorative treatments and anterior implant placement procedures in Taiwanese.
\end{abstract}

Key Words: Crown form, Gingival characteristics, Taiwanese.

\section{INTRODUCTION}

Clinically, great variation exists between humans with respect to morphological characteristics of the periodontium. It was proposed that two basic "biotypes" of gingival architecture exist, the "scalloped-thin" and the "flat-thick" types $[1,2]$. The "scalloped-thin" gingiva has been suggested to be associated with [1] tapered crown form; [2] subtle cervical convexity; and [3] minute proximal contact areas located near the incisal edge of the tooth. The "flat-thick" gingiva, on the other hand, corresponded to a tooth with [1] squared facial form; [2] distinct cervical convexity; and [3] relatively large, more apically located contact areas $[1,2]$. The severity of different symptoms associated with plaque induced periodontal lesions might be varied according to different periodontal "biotypes" $[1,2]$. Thus, a deep periodontal pocket might be the result of plaque-associated inflammation in individuals with a "flat-thick" appearance of their periodontal tissue, while individuals with a "scalloped-thin" appearance might respond with recession of the gingival margin [2]. However, most studies focused on Caucasians and rarely referred to other races. Due to the genetic variation which exists among different races, it is necessary to examine the classifications of crown forms and associated corresponding gingival characteristics in different ethnic groups.

\footnotetext{
*Address correspondence to these authors at the Department of Periodontics, Chi-Mei Medical Center, 901 Chung-Hwa Road, Yong-Kang City, Tainan, Taiwan; E-mail: jimtseng@ms15.hinet.net; and Department of Periodontics, Chung-Ho Memorial Hospital, Kaohsiung Medical University, 100 ShihChuan $1^{\text {st }}$ Road, Kaohsiung 807, Taiwan, E-mail: chchts@kmu.edu.tw
}

\section{MATERIAL AND METHODS}

This subject population consisted of 112 periodontally healthy adult Taiwanese (58 males and 54 females, aged 1929 years). Each participant gave their informed consent. Inclusion criteria for participation were as follows: (i) no medication at present or in the past 6 months including drugs known to increase the risk for gingival enlargement, such as calcium channel blockers, cyclosporine A or phenytoin; (ii) no periodontal treatment with the exception of regular prophylaxis; (iii) healthy gingivae or mild gingivitis with no periodontal probing depth in excess of $3 \mathrm{~mm}$; (iv) the free gingival margin on the facial surface of teeth in maxillary sextant positioned apically to the cervical bulge; (v) no evidence of attachment loss and periodontal probing depth within normal range; and (vi) the marginal tissue was knife edged in form, firm in consistency and coral pink in color. Subjects were excluded if (i) there was evidence of gingival alteration, (i.e. gingival overgrowth / hyperplasia, inflammation, altered passive eruption, attachment loss, gingival recession or history of periodontal surgery), or (ii) there was evidence or history of incisal edge / proximal tooth alteration i.e. (restorative intervention, traumatic injury or occlusal wear into dentine).

Subjects were provided with one session of oral hygiene instruction including the Bass technique of brushing and interdental flossing. After 1 month, a complete survey of the maxillary anterior tooth segment (central incisors, lateral incisors, canines) was performed. 


\section{CLINICAL MEASUREMENTS}

Width of keratinized gingiva (WG) is the distance measured midbuccally to the nearest $\mathrm{mm}$, from the mucogingival junction to the gingival margin using a calibrated periodontal probe : University of Michigan " $\mathrm{O}$ " probe, with Williams markings (at 1, 2, 3, 5,7, 8, 9, and 10mm) (Hu-Fridy ${ }^{\circledR}$ ).

Gingival thickness $(G T)$ at facial aspects was assessed atraumatically with a novel ultrasonic device (SDM ${ }^{\circledR}$, Krupp, Essen, Germany) to the closest $0.1 \mathrm{~mm}$. The edge of the transducer probe of $\mathrm{SDM}^{\circledR}$ was placed at a midbuccal location at the level of the periodontal probing depth. Excellent validity and reliability of the measurement device has been demonstrated elsewhere [3]. In this study, linear regression and correlation analysis were performed to describe the reproducibility of GT measurements with the ultrasonic device by the same examiner.

\section{MEASUREMENTS FOR THE DENTAL STONE MODELS}

Alginate impression of the upper jaw was taken in stock tray and poured in yellow dental stone according to manufacturer's specifications to obtain the study model. The following assessments were made (Fig. 1) according to Olsson and Lindhe on the casts, using a dial caliper (Mututoyo ${ }^{\circledR}$ Manufacturing Co. Ltd., Japan) with tips sharpened to improve access to in the interproximal areas $[4,5]$.

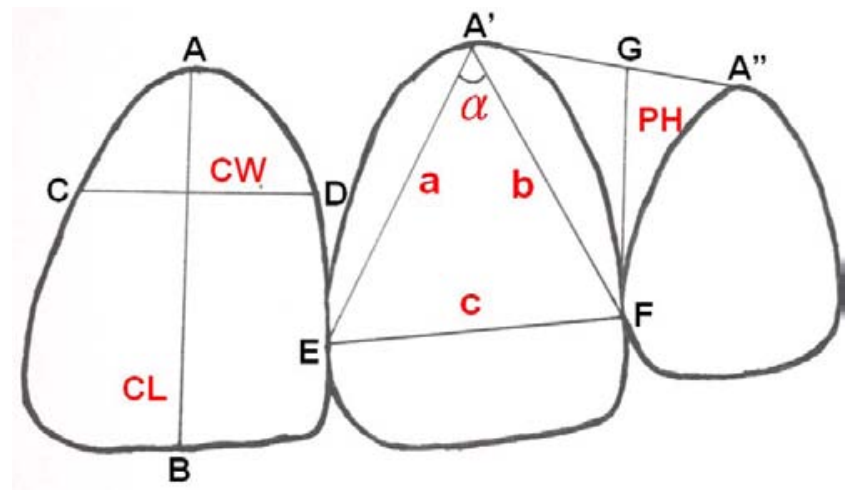

Fig. (1). A schematic illustration of measured distances of dental stone casts $(\mathrm{CL}, \mathrm{CW}, \mathrm{a}, \mathrm{b}, \mathrm{c}, \mathrm{PH}) . \mathrm{CL}=$ crown length, $\mathrm{CW}=$ crown width, $\mathrm{PH}=$ height of interdental (distal) papilla. Lines $\mathrm{a}, \mathrm{b}, \mathrm{c}$ are incorporated in the cosine formula for assessment of the angle $\alpha$.

Length of the crown $(C L)$ is the distance (AB) measured between the gingival margin (A), or if discernible, the cemento-enamel junction and the incisal edge (B) of the crown.

The CL was divided into three portions of equal height: cervical (C), middle (M) and incisal (I). The width of the crown $(C W)$ is the distance measured between the approximal tooth surfaces at the borderline between portions $\mathrm{C}$ and M.

The ratio of width and length of the crown (CW/CLratio) was calculated separately for each tooth and an individual mean value was computed for each tooth region (central incisors, lateral incisors, and canines).

The curvature of the cervical gingival margin was defined as the gingival angle (GA) that forms at the intersection between the two lines that connect the most apical portion of the buccal gingival margin with the most coronal portions of the papillae [4]. The $G A(\alpha)$ was calculated by means of the formula: $\cos \alpha=\left(a^{2}+b^{2}-c^{2}\right) / 2 a b$. The distances from the most apical portion of the gingival margin to the most coronal portions of the mesial and distal papillae are represented by $a$ and $b$ respectively in the formula. The length of the line connecting the top of the two papillae is the $c$ value in the formula (Fig. 1).

The height of the interdental papilla $(\mathrm{PH})$ determined parallel to the tooth axes is the distance ( FG ) from the top of the papilla to the line connecting the most apical points $\left(A^{\prime}, A^{\prime \prime}\right)$ of the midbuccal gingival margin of the two adjacent teeth (Fig. 1).

\section{Narrow type (group N)}

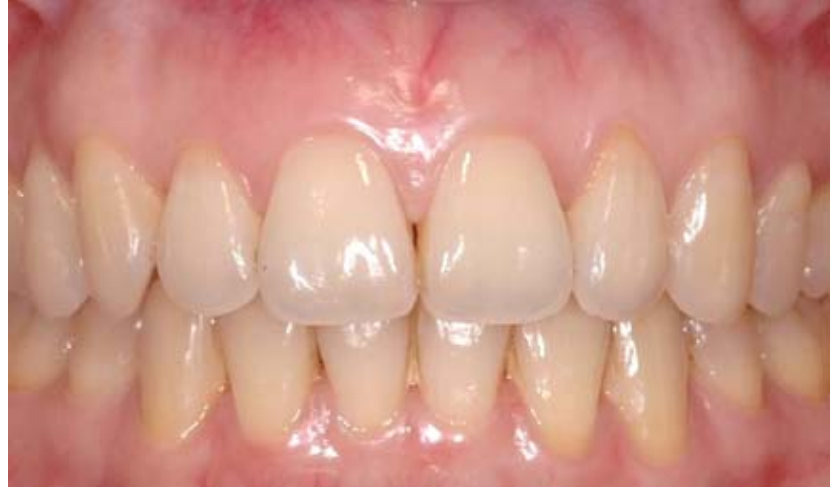

Compound type (group C)

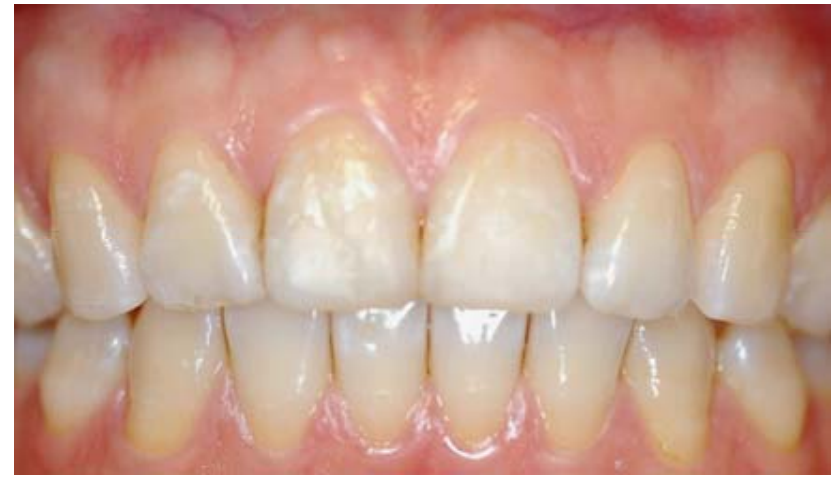

Square type (group S)

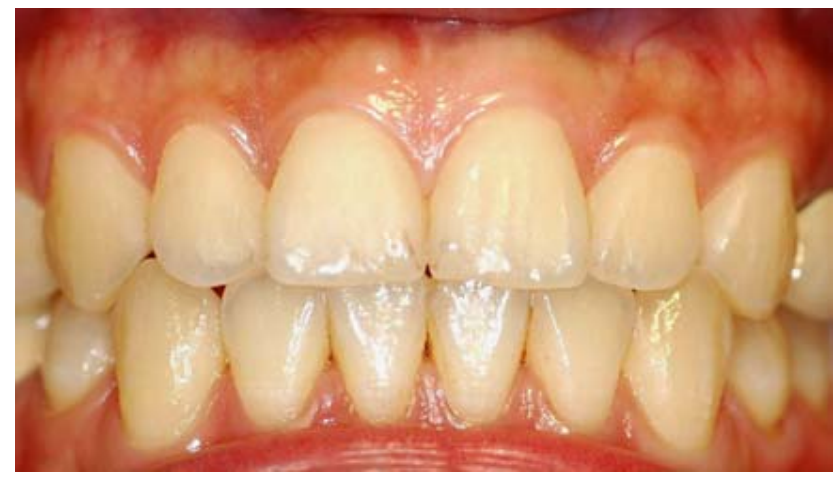

Fig. (2). Crown forms and gingival characteristics. Narrow type = Individual with a slim crown form of the upper anterior teeth. Square type = Individual with a stout crown form of the upper anterior teeth. Compound type $=$ Individual's upper anterior teeth with a crown form of CW/CL ratio between $\mathrm{N}$ and $\mathrm{S}$ types. 
Additionally, data regarding gender, age, ethnicity and subject height $(\mathrm{SH})$ were obtained from each participant.

The data for each parameter of the clinical measurements and stone models were averaged for each subject and the mean values for the subjects were compared.

Statistical analysis was carried out on one ethnic group (Taiwanese) and this was the only group represented by sufficient numbers of subjects. The mean CW, CL, CW/CLratio, GA, GT, PH and WG for each tooth group were determined by gender. Student $t$-test was used to determine the gender differences which existed between means in $\mathrm{SH}$ and above items. Pearson's correlation coefficient was used to determine correlation coefficients and their significance between means in CW, CL, CW/CL-ratio, GA, GTH, PH and $\mathrm{WG}$ of tooth groups and in $\mathrm{SH}$ within each gender group.

The influence of various parameters on the gingivae was examined by means of stepwise multiple regression, including data from the whole sample. Hence, linear models were constructed with the thickness of gingiva (GT) as the dependent variable and the individual mean CW, CL, CW/CLratio, GA, PH and WG for each tooth group as independent variables. Regression analysis was performed separately for each of the three tooth regions. A $p$-value of 0.05 was the prerequisite for a variable to be included in the models.

Cluster analysis was used to divide objectively a given population which were more easily surveyed fractions. The Euklidean distance of 9 parameters was used as a measure of distance: mean GT, WG and CW/CL-ratio of upper central incisors, lateral incisors and canines. An initial partition of the 112 subjects into 3 clusters was iteratively improved by non-hierarchical disjunctive cluster analysis with $k$-means algorithm in order to reduce the intra-group sum of squares [6]. Analyses were done on a JMP 5.0 (SAS Institute Inc., Cary, NC, USA).

\section{RESULTS}

Reproducibility of measurements was quite high in the present study. In the same examiner, for example, duplicate measurements of gingival thickness (GT) with the ultrasonic device in 10 subjects revealed that data1 $=-0.03+1.02$ data2, $\mathrm{p}<0.0001, \mathrm{r}^{2}=0.989$; correlation coefficient was 0.99 , $\mathrm{p}<0.00001$. All clinical measurements and measurements on the dental stone models were performed by one examiner (the first author).

The mean age of the subjects was 22.7 years old. The individual mean values for gingival and dental characteristics of all the samples are presented in Table $\mathbf{1}$.

We first listed the data of hard tissue and the means and standard deviations of the $\mathrm{CW}, \mathrm{CL}$, and $\mathrm{SH}$ of the three groups are presented in Table 2 . The mean CW and CL of males' central incisor and $\mathrm{CW}$ of canine were significantly greater than those of females. A statistically significant difference also existed between the means of $\mathrm{SH}$ in different genders. However, a comparison of the mean CW/CL-ratios between males and females of all 3-tooth groups wasn't significantly different.

Table 1. Mean Values and Standard Deviation for Ratios, Gingival and Dental Characteristics in all Subjects (n=112)

\begin{tabular}{|c|c|c|c|}
\hline \multirow{2}{*}{ Variable } & Central Incisor (11/21) & Lateral Incisor (12/22) & Mean \pm SD \\
\cline { 2 - 4 } & Mean \pm SD & $0.68 \pm 0.09$ & Mean \pm SD \\
\hline \hline Ratio CW / CL & $0.74 \pm 0.07$ & $5.98 \pm 0.54$ & $0.73 \pm 0.09$ \\
\hline Crown width & $7.48 \pm 0.59$ & $8.90 \pm 0.73$ & $9.86 \pm 0.57$ \\
\hline Crown length & $10.16 \pm 0.71$ & $5.74 \pm 1.30$ & $5.37 \pm 1.21$ \\
\hline Keratinized gingival width & $5.96 \pm 1.18$ & $70.65 \pm 9.15$ & $74.29 \pm 8.29$ \\
\hline Gingival angle & $70.73 \pm 6.34$ & $0.93 \pm 0.23$ & $1.01 \pm 0.27$ \\
\hline Gingival thickness & $1.23 \pm 0.35$ & $5.71 \pm 0.65$ & $5.08 \pm 0.69$ \\
\hline Papilla height & $6.65 \pm 0.81$ & & \\
\hline
\end{tabular}

Table 2. Mean (mm), Standard Deviation of Widths and Lengths of the 3- Tooth Groups of the Maxillary Dentition and Subjects, Height, by Gender

\begin{tabular}{|c|c|c|c|c|c|c|c|c|}
\hline \multirow{2}{*}{\multicolumn{2}{|c|}{ Variable }} & \multicolumn{2}{|c|}{ Central Incisor $(11 / 21)$} & \multicolumn{2}{|c|}{ Lateral Incisor $(12 / 22)$} & \multicolumn{2}{|c|}{ Canine $(13 / 23)$} & \multirow{2}{*}{ Subject Height } \\
\hline & & Width & Length & Width & Length & Width & Length & \\
\hline Male & $(\mathrm{n}=58)$ & $7.60 \pm 0.62$ & $10.30 \pm 0.67$ & $6.03 \pm 0.53$ & $8.98 \pm 0.73$ & $7.00 \pm 0.53$ & $9.54 \pm 1.03$ & $173 \pm 6.30$ \\
\hline Female & $(n=54)$ & $7.33 \pm 0.54$ & $10.01 \pm 0.71$ & $5.92 \pm 0.55$ & $8.82 \pm 0.72$ & $6.71 \pm 0.57$ & $9.47 \pm 0.81$ & $160 \pm 4.93$ \\
\hline \multicolumn{2}{|c|}{ p-value } & 0.017 & 0.03 & 0.26 & 0.26 & 0.007 & 0.68 & $<0.0001$ \\
\hline
\end{tabular}


While considering the parameters of soft tissue, we found the mean GT values (mm) of males versus females were: CI 1.3 vs. 1.15 , LI 0.96 vs. 0.9 and CA 1.07 vs. 0.94 . The mean WG values $(\mathrm{mm})$ of males versus females were: CI 5.94 vs. 5.98, LI 5.76 vs. 5.71 and CA 5.51 vs. 5.22. According to the above data, the mean GT in central incisor and canine of males were significantly greater than those of females.

Cluster analysis based on the GT, WG and CW/CL-ratio of three-tooth groups revealed three distinct appearances of crown and gingiva: narrow $(\mathrm{N})$, square $(\mathrm{S})$ and compound
(C) (Table 3). The summary statistics for levels of clinical gingival characteristics (GT and WG) and CW/CL-ratios in the three classifications are presented in Table 4 . In the narrow $(\mathrm{N})$ type, the mean GT, WG and CW/CL-ratio of upper anterior teeth were approximately $0.9 \mathrm{~mm}, 4.88 \mathrm{~mm}$ and 0.66 respectively. Besides, the sample population $(n=48 /$ 112 ) of the narrow $(\mathrm{N})$ type is more than the other two types. The compound $(\mathrm{C})$ type had the thickest $(1.27 \mathrm{~mm})$ and widest keratinized gingiva $(6.79 \mathrm{~mm})$ but a median quadratic shape (0.73) between the narrow and square group. The square $(\mathrm{S})$ type had the shortest and widest shape (0.79)

Table 3. Clinical Characteristics of Gingiva and CW/CL-Ratios (Mean and Standard Deviation) in 3 Classifications

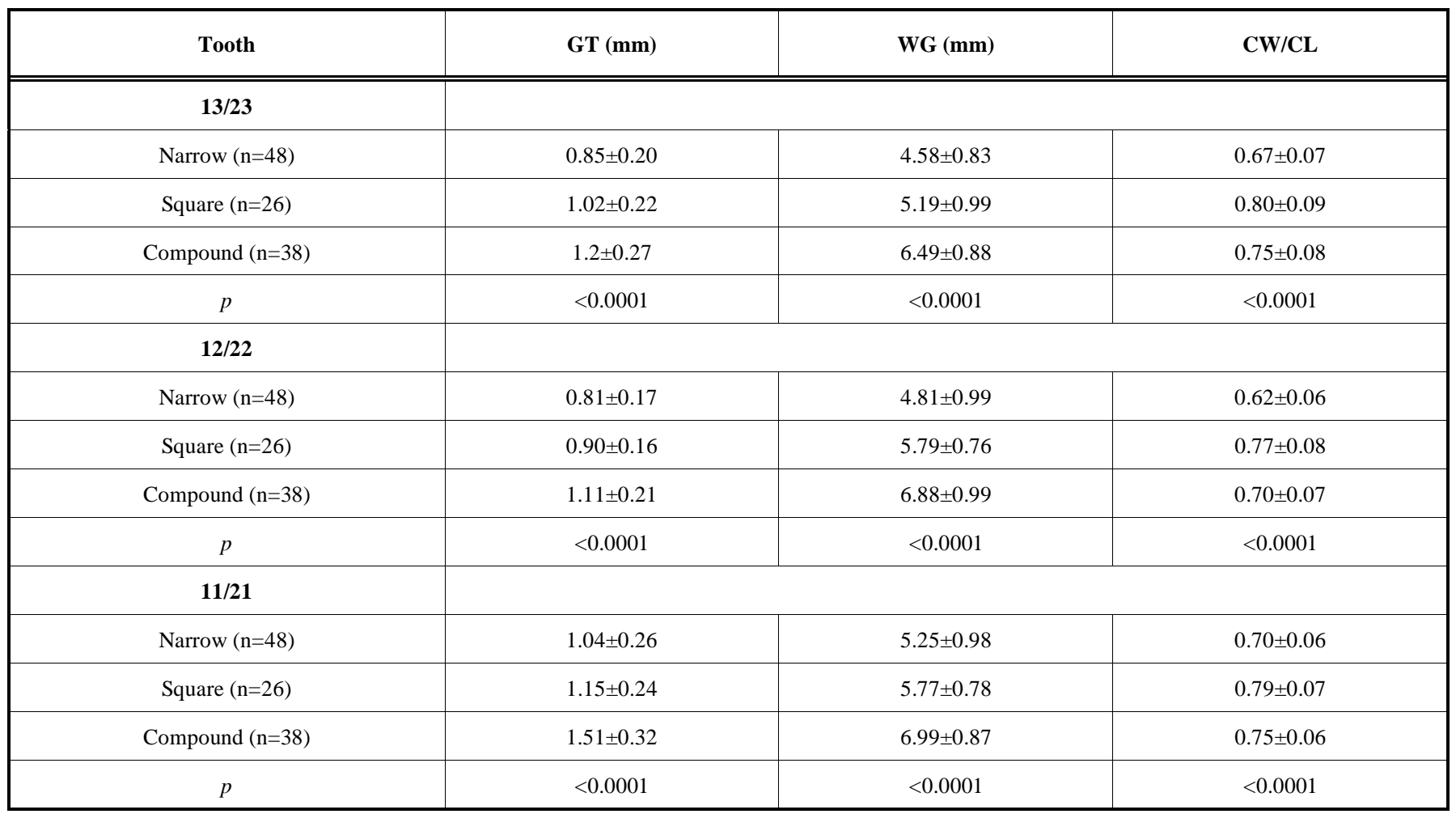

Note: Cluster analysis based on mean gingival thickness (GT), width of keratinized gingiva (WG) and ratio of crown width to crown length (CW/CL) of upper canines, lateral incisors and central incisors.

Table 4. Summary Statistics for Levels of Various Parameters Among 3 Classifications

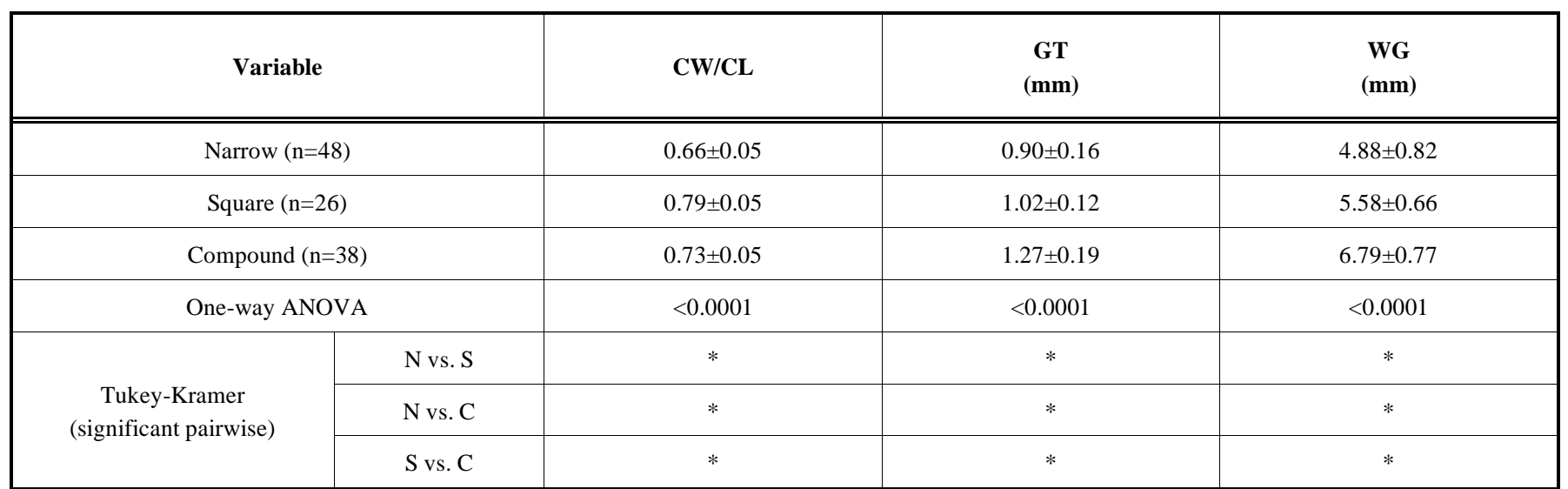

* p-value $<0.05$ by Tukey-Kramer pairwise comparison.

GT: Gingival thickness; WG: Width of keratinized gingiva; CW/CL: Crown width/Crown length -ratio. 
among three groups. However, subjects of the S type did not have the most soft tissue parameters. Instead, they had moderate GT $(1.02 \mathrm{~mm})$ and WG $(5.58 \mathrm{~mm})$ among 3 groups and the corresponding gingival values were smaller than those for the compound type. Because of the intentionally assorted subjects to clusters with similar clinical features at maxillary anterior teeth (i.e., decreasing intra-cluster sum of squares), most of the differences were highly significant.

\section{DISCUSSION}

Studies related to coronal tooth dimensions of the maxillary anterior dentition found the order of mean tooth width to be $\mathrm{CI}>\mathrm{CA}>\mathrm{LI}$ [7]. The order persists even when gender and ethnicity are accounted for [8-10]. The present study found that the order of mean crown sizes of the permanent dentition was consistent with those previously reported.

Significant gender differences in tooth dimensions of the anatomic and clinical crowns have been noted in various ethnic groups i.e. Caucasians, Afro-Americans and FrenchCaucasians $[8,10,11]$. We found that the horizontal and vertical tooth dimensions of the clinical crown for male subjects were in part significantly greater than the corresponding figures for females in Taiwanese.

Filipsson \& Goldson found no correlation between subject standing height $(\mathrm{SH})$ and tooth size [12]. On the other hand, Garn found a "low-order relationship" between the two variables [13]. We found no correlation between $\mathrm{CW} / \mathrm{CL}-$ ratios and $\mathrm{SH}$ in three-tooth groups among Taiwanese.

However, in the present study, all Taiwanese subjects yielded lower values of CW/CL-ratio among all 3-tooth groups than the mean value obtained in the studies of Sterrett [14] factors in; It implied that crown forms in Taiwanese seemed more slender than those in Caucasian and Taiwanese might expose labial gingival recession. In other words, Taiwanese are more likely to display longer teeth with dark triangle. Therefore, it is a challenge to reconstruct maxillary anterior teeth esthetically.

The term periodontal biotype used to designate distinct features ("flat-thick" or "scalloped-thin") of the periodontium including the underlying alveolar bone was proposed by Seibert \& Lindhe [2]. In the current study, however, the more customary term phenotype is used to describe the features of the marginal periodontium that are influenced by both genetic and environmental factors [14]. According to the analysis of covariance, the subject was an important factor determining GT [3], it was presumed that there exist groups with different crown forms with corresponding gingival characteristics. Since cluster analysis is an interactive process, a number of different solutions were evaluated. Initially, the studied subjects categorized into three crown-gingival classifications (narrow, square and compound) seemed to be the most reasonable and was iteratively improved by nonhierarchical disjunctive cluster analysis. In our studied subjects, $43 \%$ were in the narrow $(\mathrm{N})$ group with the thinnest gingival thickness, the minimal width of keratinized gingiva and the slimmest crown shape; $23 \%$ were in the square (S) group with similar gingival thickness, medium width of keratinized gingiva and the stoutest form of upper anterior teeth; and $34 \%$ were in the compound (C) type with the bulkiest gingival thickness, the amplest width of keratinized gingiva at facial aspects and mid CW/CL-ratio.

Olsson et al. (1993) reported significantly wider keratinized tissue at facial aspects, a lower papilla height, a higher gingival angle of the crown, but no significant difference in GT in short-wide as compared with long-narrow central incisors [5]. Eger et al. (1996) also failed to observe a meaningful influence of CW/CL-ratio with GT [3]. Obviously, there were at least two different phenotypes being associated with a wide-short type of crown in the upper anterior segment. In this context, Muller (1997) found two subgroups of wideshort shape of maxillary front dentition: one with a "normal" gingival thickness and narrow band of keratinized tissue, and the other with thick and wide keratinized gingiva among 42 male Caucasian subjects [15]. The present findings suggest that there are both different phenotype combinations in clinical periodontal appearance for Caucasians and Taiwanese: square (S) and compound (C) classifications. And the above data based on clinical evidence seemed to contradict the hypothesis of Seibert and Lindhe [2]. There are not only two biotypes, but also three classifications of crown forms and gingival characteristics. We could obtain the gingival thickness of a tooth by calculating the CW/CL-ratio and width of keratinized gingiva without transgingival probing. According to the above evidence, it's suspected that subjects with square type crown form display gingival recession even if they had the most wide-short crown forms; in contrast, subjects with compound type crown form might have deep probing depths in the future. Therefore, we should offer different treatments to subjects with different clinical classifications. For patients with narrow $(\mathrm{N})$ and square $(\mathrm{S})$ crown form, due to thinner gingival thickness, bilaminar subepithelial connective tissue graft [16-19] or free gingival onlay graft were utilized [20-24] to prevent recession or dark color from gingiva while they received implant treatment or crown restoration. In compound type subjects, coronal or original positioned flap during implant placement met esthetic criteria due to thicker gingival thickness.

With genetic polymorphisms, it would be of some interest to extend these investigations to confirm the existence of the proposed crown-gingival classifications and to determine the prevalence in different gender, physical constitutional and racial groups. In addition, as for subjects with different types, we should take various treatment modalities into consideration.

\section{ACKNOWLEDGEMENTS}

The author extends sincere thanks to the Departments of Periodontics and Prosthodontics, Graduate Institute of Dental Sciences, College of Dental Medicine, Kaohsiung Medical University, Taiwan, for their valuable advises. We also thank Chi Mei Medical Center, Tainan, Taiwan and Kaohsiung Medical University for the grant support.

\section{REFERENCES}

[1] Weisgold AS. Contours of the full crown restoration. Alpha Omegan 1977; 70: 77-89.

[2] Seibert J, Lindhe J. Esthetics and Periodontal Therapy, 2nd ed. Copenhagen: Munksgaard 1989.

[3] Eger T, Muller HP, Heinecke A. Ultrasonic determination of gingival thickness. Subject variation and influence of tooth type and clinical features. J Clin Periodontol 1996; 23:839-45. 
[4] Olsson M, Lindhe J. Periodontal characteristics in individuals with varying form of the upper central incisors. J Clin Periodontol 1991; 18: 78-82.

[5] Olsson M, Lindhe J, Marinello CP. On the relationship between crown form and clinical features of the gingiva in adolescents. $J$ Clin Periodontol 1993; 20: 570-7.

[6] Hartigan JA, Wong MA. A k-means clustering algorithm. Appl Stat 1979; 28:100-8.

[7] Ballard ML. Asymmetry in tooth size: a factor in the etiology, diagnosis and treatment of malocclusion. Angle Orthod 1944; 14 : 67-70.

[8] Lavelle CL. Maxillary and mandibular tooth size in different racial groups and in different occlusal categories. Am J Orthod 1972; 61: 29-37.

[9] Doris JM, Bernard BW, Kuftinec MM, Stom D. A biometric study of tooth size and dental crowding. Am J Orthod 1981; 79: 326-36.

[10] Gillen RJ, Schwartz RS, Hilton TJ, Evans DB. An analysis of selected normative tooth proportions. Int J Prosthodont 1994; 7: 41017.

[11] Howe RP, McNamara JA, Jr., O'Connor KA. An examination of dental crowding and its relationship to tooth size and arch dimension. Am J Orthod 1983; 83: 363-73.

[12] Filipsson R, Goldson L. Correlation between tooth width, width of the head, length of the head, and stature. Acta Odontol Scand 1963; 21: 359-65.

[13] Garn SM, Lewis AB, Kerewsky RS. The magnitude and implications of the relationship between tooth size and body size. Arch Oral Biol 1968; 13: 129-31.

[14] Sterrett JD, Oliver T, Robinson F, Fortson W, Knaak B, Russell CM. Width/length ratios of normal clinical crowns of the maxillary anterior dentition in man. J Clin Periodontol 1999; 26: 153-7.
[15] Muller HP, Eger T. Gingival phenotypes in young male adults. J Clin Periodontol 1997; 24: 65-71.

[16] Kan JY, Rungcharassaeng K, Lozada JL. Bilaminar subepithelial connective tissue grafts for immediate implant placement and provisionalization in the esthetic zone. J Calif Dent Assoc 2005; 33: 865-71.

[17] Shibli JA, d'avila S, Marcantonio E Jr. Connective tissue graft to correct peri-implant soft tissue margin: A clinical report. . J Prosthet Dent 2004; 91: 119-22

[18] Silverstein LH, Lefkove MD. The use of the subepithelial connective graft to enhance both the aesthetics and periodontal contours surrounding dental implants. J Oral Implantol 1994; 20:135-8.

[19] Price RB, Price DE. Esthetic restoration of a single-tooth dental implant using a subepithelial connective graft: a case report with 3 year follow. Int J Periodontics Restorative Dent 1999; 19: 92-101.

[20] El Askary AS. Use of connective tissue grafts to enhance the esthetic outcome of implant treatment: a clinical report of 2 patients. J Prosthet Dent 2002; 87: 129-32.

[21] Sevor JJ. The use of free gingival graft to improve the implant soft tissue interface: rationale and technique. Pract Periodontics Aesthet Dent 1992; 4: 59-64.

[22] Horning GM, Mullen MP. Peri-implant free gingival grafts: rationale and technique. Compend Contin Educ Dent 1990; 11: 604, 60610 .

[23] Simons AM, Darany DG, Giordano JR. The use of free gingival graft in the treatment of peri-implant soft tissue complications: clinical report. Implant Dent 1993; 2: 27-30.

[24] Silverstein LH, Lefkove MD, Garnick JJ. The use of free gingival soft tissue to improve the implant/soft-tissue interface. J Oral Implantol 1994; 20: 36-40.

(C) Chou et al.; Licensee Bentham Open.

This is an open access article licensed under the terms of the Creative Commons Attribution Non-Commercial License (http://creativecommons.org/licenses/ by-nc/3.0/) which permits unrestricted, non-commercial use, distribution and reproduction in any medium, provided the work is properly cited. 